\title{
A Linear Active Disturbance Rejection Control for a Ball and Rigid Triangle System
}

\author{
Carlos Aguilar-Ibanez, ${ }^{1}$ Hebertt Sira-Ramirez, ${ }^{2}$ and Miguel S. Suarez-Castanon ${ }^{3}$ \\ ${ }^{1}$ CIC, Instituto Politécnico Nacional, Ciudad de México, DF, Mexico \\ ${ }^{2}$ Sección de Mecatrónica, CINVESTAV, Ciudad de México, DF, Mexico \\ ${ }^{3}$ ESCOM, Instituto Politécnico Nacional, Ciudad de México, DF, Mexico
}

Correspondence should be addressed to Carlos Aguilar-Ibanez; carlosaguilari@cic.ipn.mx

Received 6 May 2016; Revised 5 September 2016; Accepted 15 September 2016

Academic Editor: Alessio Merola

Copyright (C) 2016 Carlos Aguilar-Ibanez et al. This is an open access article distributed under the Creative Commons Attribution License, which permits unrestricted use, distribution, and reproduction in any medium, provided the original work is properly cited.

\begin{abstract}
This paper proposes an application of linear flatness control along with active disturbance rejection control (ADRC) for the local stabilization and trajectory tracking problems in the underactuated ball and rigid triangle system. To this end, an observer-based linear controller of the ADRC type is designed based on the flat tangent linearization of the system around its corresponding unstable equilibrium rest position. It was accomplished through two decoupled linear extended observers and a single linear output feedback controller, with disturbance cancelation features. The controller guarantees locally exponentially asymptotic stability for the stabilization problem and practical local stability in the solution of the tracking error. An advantage of combining the flatness and the ADRC methods is that it possible to perform online estimates and cancels the undesirable effects of the higher-order nonlinearities discarded by the linearization approximation. Simulation indicates that the proposed controller behaves remarkably well, having an acceptable domain of attraction.
\end{abstract}

\section{Introduction}

In the last 3 decades, there has been increasing interest in the control of underactuated mechanical systems. These systems are characterized by having fewer actuators than degrees of freedom [1]. This class of mechanical systems is becoming popular in many control applications, such as spacecraft, aerial robotic systems, underwater vehicles, and flexible robotics, to mention a few [2-4]. Possible advantages associated with these systems are cost reduction, lighter structures, and smaller dimensions, among others (see [57] for a comprehensive treatment of this class of systems). In this context, we are interested in the output feedback asymptotic stabilization and the output feedback trajectory control problems of the uncertain ball and rigid triangle (BRT) system. This system consists of a rigid isosceles triangle, $O A B$, hung from the vertex, $O$, and able to rotate around this vertex by the action of the torque, $\tau$ (see Figure 1). The identically rigid beams, $\overline{O A}$ and $\overline{O B}$, of mass $m_{0}$ and length $R$, are attached to the left and right ends, respectively, of a rigid homogeneous beam, $\overline{A B}$, of length $2 L$ and mass $M$. Over the $\overline{A B}$ beam, there is a ball of mass $m$, which can move freely forwards and backwards on a direction parallel to it. As is evident, this ball moves when the triangle rotates and the $\overline{A B}$ beam bends either side. As the figure shows, the system is quite nonlinear due to the gravitational forces, the Coriolis and centripetal forces, and the acceleration couplings. On the other hand, by simple comparison, it is easy to see that this system is quite similar to but more complex than the traditional ball and beam system [6], because both dynamics of this system are completely coupled. Therefore, this system is not feedback-linearizable. In addition, it does not have a well defined relative degree [8].

Fortunately, the linearized tangent model of this system is locally controllable around the unstable equilibrium point, meaning that it is locally flat. Hence, the stabilization and tracking problems both can be solved locally, from a combined perspective of flatness approach and active 


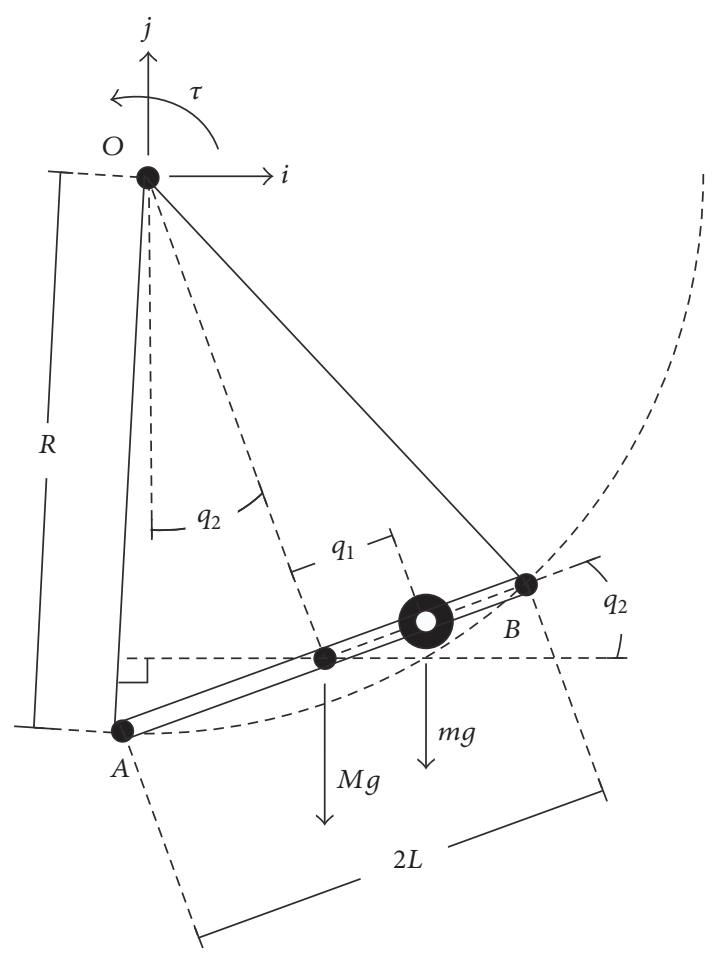

FIGURE 1: The ball and rigid triangle system.

disturbance rejection control (ADRC). To accomplish this, we can propose two decoupled extended linear observers, assuming that only the position variables of this system are available. These observers allow us to simultaneously estimate the time derivatives of the nonavailable flat output and recover the uncertain underlying nonlinear dynamics. Subsequently, these estimations, together with the ADRC approach, allow us to propose a control scheme to solve the aforementioned control problems. The main difference between flatness controllers [9-12] and the ADRC scheme for flat systems is the fact that traditional flatness-based controls need perfect knowledge of the plant, while ADRC schemes for flat systems may largely ignore unknown nonlinearities and exogenous additive perturbation inputs in the input-toflat output dynamics $[13,14]$. We underscore that the brilliant idea of designing a controller with the capacity of lumped compensation of endogenous and exogenous disturbances, by means of an observer-based control, was proposed by Han [15]. He was the first to introduce the concept of the ADRC. ADRC controllers have led to new paradigmatic traditional nonlinear control problems in which disturbances, internal and external, are actively estimated and rejected $[16,17]$. This concept has been used lately for controlling some kinds of underactuated systems in conjunction with the differential flatness approach with promising results (see [14, 18-22]). Recall that we can use this approach for systems in which tangent linear approximation is locally controllable.

In this article, an ADRC scheme with a flatness-based approach is proposed to practically solve the output feedback stabilization and the output feedback trajectory tracking problem for the BRT system. Our control approach assumes a lack of knowledge of the system parameters, the nonlinearities, and exogenous disturbance signals. The scheme not only estimates the unknown dynamics and the unknown state variables, but also reduces the tracking control problem to that defined on a chain of integrators after online active disturbance cancelations. Finally, the control algorithm is tested in several numerical simulations, showing excellent results for the output stabilization problem and output tracking problem. It is worth mentioning that the basin idea of this methodology is sustained by the use of high-gain observers, which are used as identifiers of the uncertain dynamics, assuming that in some operational region this uncertainty can be considered as being smooth and bounded. This kind of methodology has been used in [23-26].

The rest of this study is organized as follows. In Section 2, we obtain the nonlinear model of the BRT system by using the Euler Lagrange method. We normalize the obtained nonlinear model; then we linearize it around to its unstable equilibrium, and we show that the incremental linear model is flat with respect to a suitable output. In Section 3, we briefly describe the ADRC approach and introduce a useful theorem related to its stability. In Section 4, we propose the two decoupled extended observers and design a suitable ADRC controller. The results of the numerical simulations showing the effectiveness of our proposal are presented in Section 5, while Appendix is devoted to final remarks and future work.

Notation. In the forthcoming developments, we use the following configuration:

$$
\begin{aligned}
& \dot{x}=A_{i} x+E_{i} u \\
& y=C_{i}^{T} x
\end{aligned}
$$

where $x \in \mathbb{R}^{i}$ denotes the system state, $u \in \mathbb{R}$ is the system single input, and matrices $A_{i} \in \mathbb{R}^{i \times i}, E_{i} \in \mathbb{R}^{i}$, and $C_{i} \in \mathbb{R}^{i}$ are defined according to the well-known Brunovsky form. Finally, $\lambda_{i}\{X\}$ stands for the eigenvalues of matrix $X$.

\section{The Ball and Rigid Triangle (BRT) Model}

Obtaining the Mathematical Model. We first consider a fixed reference frame, located at the fixed point $O$, with the horizontal and vertical unidirectional vectors, given by $\vec{i}$ and $\vec{j}$, respectively (see Figure 1). The generalized coordinates are denoted by the vector $q=\left[q_{1}, q_{2}\right]$. The coordinate $\left|q_{1}\right|<L$ is the cart position, measured from itself to the gravity center of the beam. The coordinate $\left|q_{2}\right|<\pi / 2$ is the angle formed by the vertical line passing through $O$ and the imaginary line passing through $O$ and the beam gravity center. To simplify the dynamical model and capture its nonlinear nature, the masses of $\overline{O A}$ and $\overline{O B}$ beams are considered equal to zero. Evidently, the distance between the fixed point $O$ and the gravity center of the $\overline{A B}$ beam is $L_{R}=\sqrt{R^{2}-L^{2}}$. After some physical considerations, it is easy to show that the kinetic energy of the triangle structure is defined as

$$
K_{M}(\dot{q})=\frac{1}{2} I_{b} \dot{q}_{2}^{2}+\frac{1}{2} M L_{R}^{2} \dot{q}_{2}^{2}
$$


where $I_{b}$ is the total moment of inertia of the $\overline{A B}$ beam. Similarly, the kinetic energy related to the mass, $m$, is

$$
K_{m}(q, \dot{q})=\frac{1}{2} m\left(L_{R}^{2} \dot{q}_{2}^{2}+q_{1}^{2} \dot{q}_{2}^{2}+2 L_{R} \dot{q}_{1} \dot{q}_{2}+\dot{q}_{1}^{2}\right)
$$

Therefore, the total kinetic energy of the system is

$$
K(q, \dot{q})=K_{m}(q, \dot{q})+K_{M}(\dot{q})
$$

The potential energies associated with the beam and the moving mass are, respectively, given by

$$
\begin{aligned}
& P_{m}(q)=M g\left(1-\cos q_{2}\right) ; \\
& P_{m}(q)=m g\left(L_{R}\left(1-\cos q_{2}\right)+q_{1} \sin q_{2}\right) .
\end{aligned}
$$

In addition, the total potential energy of the system is defined as

$$
P(q, \dot{q})=P_{m}(q)+P_{M}(q)
$$

From (4) and (6), we can write the Lagrangian function as

$$
L(q, \dot{q})=K(q, \dot{q})-P(q) .
$$

Therefore, the Euler Lagrange equations are

$$
\begin{aligned}
& m \ddot{q}_{1}+m R_{0} \ddot{q}_{2}-m q_{1} \dot{q}_{2}^{2}+m g \sin q_{2}=0 ; \\
& m R_{0} \ddot{q}_{1}+I(q) \ddot{q}_{2}+2 m q_{1} \dot{q}_{1} \dot{q}_{2}+(M+m) g R_{0} \sin q_{2} \\
& \quad+m g q_{1} \cos q_{2}=\tau,
\end{aligned}
$$

where

$$
I(q)=\left(I_{b}+m L_{R}^{2}\right)+m q_{1}^{2}
$$

To simplify the algebraic manipulations in the forthcoming developments, the above equations are normalized using the following transformations:

$$
\begin{aligned}
& x_{1}=\frac{q_{1}}{L_{R}} ; \\
& x_{2}=q_{2} ; \\
& u=\frac{\tau}{m g} ; \\
& \epsilon=t \sqrt{\frac{g}{L_{R}}},
\end{aligned}
$$

leading to the following simpler system:

$$
\begin{aligned}
& \ddot{x}_{1}+\ddot{x}_{2}-x_{1} \dot{x}_{2}^{2}+\sin x_{2}=0 ; \\
& \ddot{x}_{1}+\left(1+x_{1}^{2}+N\right) \ddot{x}_{2}+2 x_{1} \dot{x}_{1} \dot{x}_{2}+(1+\mu) \sin x_{2} \\
& \quad+x_{1} \cos x_{2}=u,
\end{aligned}
$$

with $N=I_{b} /\left(m L_{R}^{2}\right)$ and $\mu=M / m$, where "." stands for differentiation with respect to the dimensionless $\epsilon$.
Evidently, the system above admits the following EulerLagrange representation:

$$
M_{n}(x) \ddot{x}+C_{n}(x, \dot{x}) \dot{x}+G_{n}(x)=U \text {, }
$$

where $x=\left(x_{1}, x_{2}\right)$ and

$$
\begin{aligned}
M_{n}(x) & =\left[\begin{array}{cc}
1 & 1 \\
1 & 1+x_{1}^{2}+N
\end{array}\right] ; \\
G_{n}(x) & =\frac{\partial}{\partial x} V_{n}(x) ; \\
C_{n}(x, \dot{x}) & =\left[\begin{array}{cc}
0 & x_{1} \dot{x}_{2} \\
x_{1} \dot{x}_{2} & x_{1} \dot{x}_{1}
\end{array}\right] ; \\
U & =\left[\begin{array}{l}
0 \\
u
\end{array}\right],
\end{aligned}
$$

and $V_{n}(x)=-(1+\mu) \cos x_{2}+x_{1} \sin x_{2}$. Finally, it is easy to see that system (8) verifies the following properties: (i) $M_{n}(x)>0$; (ii) the matrix $S=M_{n}(x)-2 C_{n}(x, \dot{x})$ is skew symmetric; and (iii) the operator $u \rightarrow \dot{x}_{2}$ is passive; that is, the energy function time derivative $\dot{E}_{n}=\dot{x}_{2} u$, where $E_{n}=\dot{x}^{T} M_{n}(x) \dot{x} / 2+V_{n}(x)$ (notice that the sign of $E_{n}$ is not well defined).

Problem Statement. Given the system model described in (8), the objective of this work consists of two goals: (1) designing a linear stabilizing controller to simultaneously bring the rigid triangle and the cart to the zero position $\left(x_{1}=x_{2}=\right.$ 0 ), assuming that all variables are initialized within a small vicinity of the origin, and (2) solving locally the output feedback trajectory tracking problem.

Motivation. It is easy to see that system (11) is unstable around the zero equilibrium point. In addition, this system cannot be feedback-linearizable using either static or dynamic state feedback. This fact is easy to verify by simply comparing the structure of the BRT system with the structure of the complete model of the ball and beam system [6]. On the other hand, we strongly believe that the problem of stabilizing the system (11) has not been solved, until now, using the energy shape based control method (see [27-29]). Additionally, there has been no analytical expression for the closed loop energy function. Here, we provide an approximated control solution by considering a linear version of the original model of the BRT system, in conjunction with the ADRC approach. In our opinion, this mechanical system can be applied to balance loads. For instance, the model can be extended to a three-dimensional case, which represents a container inside which a heavy object can slide freely due to load and unload maneuvers, where we want to prevent the object from hitting and damaging. Finally, we mention that this model can be considered as a simplification of the ball and plate system or the ball and beam system if the radius ball is considered in the model [30, 31]. 
2.1. Flatness of the Linearized Model. Consider the tangent linearization of system (11) around the following unstable equilibrium points:

$$
\left(x_{1}=0, \dot{x}_{1}=0, x_{2}=0, \dot{x}_{2}=0, u=0\right) .
$$

This leads to the following linearized system:

$$
\begin{array}{r}
\ddot{x}_{1 \delta}+\ddot{x}_{2 \delta}+x_{2 \delta}=0 ; \\
\ddot{x}_{1 \delta}+(1+N) \ddot{x}_{2 \delta}+(1+\mu) x_{2 \delta}+x_{1 \delta}=u_{\delta},
\end{array}
$$

where $x_{1 \delta}=x_{1}$ and $x_{2 \delta}=x_{2}$ are the incremental states of the linearized system. Then, the incremental flat output, $F_{\delta}$, is

$$
F_{\text {delta }}=x_{1 \delta}+x_{2 \delta} .
$$

Indeed, all variables can be parameterized in terms of $F_{\delta}$ and a finite number of its time derivatives, as follows:

$$
\begin{aligned}
& x_{1 \delta}=F_{\delta}+F_{\delta}^{(2)} ; \\
& x_{2 \delta}=-F_{\delta}^{(2)} ; \\
& \dot{x}_{1 \delta}=F_{\delta}^{(1)}+F_{\delta}^{(3)} ; \\
& \dot{x}_{2 \delta}=-F_{\delta}^{(3)} ; \\
& u_{\delta}=-F_{\delta}^{(4)} N+x_{1}+\mu x_{2} .
\end{aligned}
$$

2.2. A Flatness-Based Pole Placement Approach for Stabilization. The state-dependent input-coordinate transformation,

$$
u_{\delta}=-v_{\delta} N+x_{1}+\mu x_{2},
$$

shows that system (15) is equivalent to the following chain of integrators:

$$
F_{\delta}^{(4)}=v_{\delta}
$$

where $v_{\delta}$ is the new stabilizing feedback controller, fixed as

$$
v_{\delta}=F_{*}^{(4)}-\sum_{i=0}^{4} k_{i}\left(F_{\delta}^{(i)}-F_{*}^{(i)}\right),
$$

where the set of coefficients $\left\{k_{1}, k_{2}, k_{3}, k_{4}\right\}$ is selected, such that the closed loop characteristic polynomial,

$$
p(s)=s^{4}+k_{4} s^{3}+k_{3} s^{2}+k_{2} s+k_{1}
$$

is Hurwitz, and $F_{*}=F_{*}(t)$ is the desired reference signal, with bounded time derivatives up to order 4 . For a detailed treatment of the flatness approach, we suggest $[9,11]$.

Remark 1. Recall that the time derivatives $F_{\delta}^{(i)}, i=\{0,1,2,3\}$ are given in the original coordinates as

$$
\begin{aligned}
& F_{\delta}=x_{1 \delta}+x_{2 \delta} ; \\
& F_{\delta}^{(1)}=\dot{x}_{1 \delta}+\dot{x}_{2 \delta} ; \\
& F_{\delta}^{(2)}=-x_{2 \delta} ; \\
& F_{\delta}^{(3)}=-\dot{x}_{2 \delta} .
\end{aligned}
$$

In the following section, we develop a control strategy for the BRT system, based on the ADRC.
Numerical Simulation. To show the closed loop response of the nonlinear normalized system (11) to the derived flatness controller (18), two simulations were carried out. To this end, the physical parameters were fixed as

$$
\begin{aligned}
m & =0.1 \mathrm{Kg} ; \\
M & =0.5 \mathrm{Kg} ; \\
L_{r} & =0.5 \mathrm{~m} ; \\
L & =0.5 \mathrm{~m} ; \\
I_{b} & =0.13 \mathrm{Kgm}^{2},
\end{aligned}
$$

while the closed loop characteristic polynomial was chosen to be

$$
p(s)=\left(s^{2}+2 \zeta \omega_{n} s+\omega_{n}^{2}\right)^{2} .
$$

\section{ADRC to Locally Control the Ball and Rigid Triangle (BRT) System}

In this section, we solve once again the previously mentioned control problem, assuming that only the position is available for measurements and that some dynamics of the original system are unknown. Before developing our control strategy, we give a summary on the ADRC subject.

3.1. Summary of $A D R C$. Let us consider the uncertain plant of $n$ th-order, described by the following set of nonlinear differential equations:

$$
\begin{aligned}
& \dot{\mathbf{x}}=A_{n} \mathbf{x}+E_{n}(b u+f(\mathbf{x}, d)) ; \\
& y=C_{n}^{T} \mathbf{x},
\end{aligned}
$$

where $\mathbf{x}=\left[x_{1}, x_{2}, \ldots, x_{n}\right]^{T} \in \mathbb{R}^{n}$ is the state; $u$ and $y \in \mathbb{R}$ are, respectively, the input and output; $b$ is a known constant; $d$ is the external unknown disturbance; and $f(\cdot)$ stands for the unknown nonlinear time-varying dynamics. Next, consider the following extended observer (EOS) for system (25):

$$
\begin{aligned}
& \dot{\widehat{\mathbf{x}}}_{e}=A_{n+1} \widehat{\mathbf{x}}_{e}+B_{n+1} u+l(y-\widehat{y}) ; \\
& \widehat{y}_{e}=C_{n+1}^{T} \widehat{\mathbf{x}}_{e},
\end{aligned}
$$

where $B_{n}^{T}=[0,0, \ldots, 1,0] \in \mathbb{R}^{n}$, and the estimation vector $\widehat{\mathbf{x}}_{e} \in \mathbb{R}^{n+1}$ is defined as

$$
\widehat{\mathbf{x}}_{e}:=\left[\widehat{x}_{1}, \widehat{x}_{2}, \ldots, \widehat{x}_{n}, \widehat{x}_{n+1}\right]^{T}=[\widehat{\mathbf{x}}, \widehat{f}]^{T},
$$

and $l$ is the gains vector of the observer, set as

$$
l=\left[w_{0} \alpha_{1}, w_{0}^{2} \alpha_{2}, \ldots, w_{0}^{n+1} \alpha_{n+1}\right]^{T},
$$

where $w_{0}>0$, with constants $\alpha_{i}$ defined as

$$
\alpha_{i}:=\frac{(n+1) !}{i !(n+1-i) !} ; \quad i=\{1,2, \ldots, n+1\} .
$$


According to this fact, it is clear that the matrix,

$$
\underline{A}=\left[\begin{array}{ccccc}
-\alpha_{1} & 1 & 0 & \cdots & 0 \\
-\alpha_{2} & 0 & 1 & \cdots & 0 \\
\vdots & \vdots & \vdots & \cdots & 0 \\
\vdots & \vdots & & \cdots & 1 \\
-\alpha_{n+1} & 0 & & \cdots & 0
\end{array}\right]
$$

is Hurwitz.

Theorem 2. Consider system (25), in closed loop with

$$
u=-\frac{1}{b}\left(K^{T}\left(\widehat{\mathbf{x}}-\mathbf{y}_{r}\right)+\widehat{x}_{n+1}-y_{r}^{(n)}\right)
$$

where $\widehat{\mathbf{x}}=\left[\widehat{x}_{1}, \ldots, \widehat{x}_{n}\right]^{T}$ and $\widehat{x}_{n+1}$ are computed via (26) and (27), with $K \in R^{n}$ selected, such that

$$
H_{n}=A_{n}-E_{n} K^{T}
$$

is a Hurwitz matrix, and $\mathbf{y}_{r}^{T}=\left[y_{r}, \dot{y}_{r}, \ldots, y_{r}^{(n-1)}\right]$ is the reference vector, with $\left|y_{r}^{(j)}\right| \leq \Gamma_{j}$, for $j=\{0, \ldots, n+1\}$, provided that

(A1) $\nabla_{\mathbf{x}} f$ and $f_{d}=\partial f / \partial d$ are bounded within the domain of interest, with $d, \dot{d} \in L_{\infty}$, and $f(x, d)$ being continuous;

(A2) $\lambda_{i}\left\{H_{n}\right\} \neq \lambda_{j}\left\{w_{0} \underline{A}\right\}$, for all $i$ and $j$, where $i=$ $\{1,2, \ldots, n\}$, and $j=\{1,2, \ldots, n+1\}$;

(A3) $w_{0}>\max \left\{1,\left\|H_{n}\right\|\left\|\underline{A}^{-1}\right\|\right\}$. Then, the output feedback dynamic, defined by (26) and (31), is practically stable. Moreover, $\widetilde{\mathbf{x}}_{e}=\mathbf{x}_{e}-\widehat{\mathbf{x}}_{e}$ is bounded with the ultimate bound given by $\mathrm{O}\left(1 / w_{0}\right)$, where $\mathbf{x}_{e}=[\mathbf{x}, f]^{T}$. Finally, if $\Delta(t)=0$, where,

$$
\Delta(t)=f_{d} \dot{d}+\frac{\partial f}{\partial x_{n}} y_{r}^{(n)}+\left(\nabla_{\mathbf{x}} f\right) A_{n} \mathbf{y}_{r},
$$

then $\widetilde{\mathbf{x}}_{e}$ exponentially converges to zero. The proof of this theorem can be found in Appendix.

\section{A GPI Observer-Based Active Disturbance Rejection Controller}

From the linearized control model (15), we use the following simplified perturbed model for the unknown nonlinear system (11), defined as

$$
F_{\delta}^{(4)}=-\frac{u_{\delta}}{N}+\eta(t)
$$

where $\eta(t)$ acts in place of all the following: the state dependent expressions, all the higher-order terms (h.o.t.) neglected by the linearization, possibly of nonmodeled dynamics, and, finally, the external unknown disturbances affecting the system. All of these uncertain terms are grouped together as a single time-varying function, which in our case has the following form:

$$
\eta(t)=\frac{1}{N}\left(F_{\delta}+F_{\delta}^{(2)}\right)-\frac{\mu}{N} F_{\delta}^{(2)}+\text { h.o.t. }
$$

Assuming that $\eta(t)$ satisfies Assumption (A1) found in Theorem 2, it is possible to solve the output feedback trajectory control problem of the uncertain system (34). That is, we can propose an admissible reference trajectory, $F_{*}=F_{*}(t)$, in order to carry out some controlled maneuver tasks. Evidently, this trajectory has to be chosen, such that it is very close to the rest position $\left(x_{1 \delta}=0, x_{2 \delta}=0\right)$. Recall that the incremental linear control model (34) is valid, as long as the trajectories of the original system are in a vicinity close to the origin. In order to solve this problem, we defined the trajectory tracking control errors as

$$
e_{i}=F_{\delta}^{(i-1)}-F_{*}^{(i-1)}(t), \quad \text { with } i=\{1,2,3,4\} .
$$

Based on this definition, we can see that the errors evolve to

$$
\begin{aligned}
& \dot{e}_{1}=e_{2} ; \\
& \dot{e}_{2}=e_{3} ; \\
& \dot{e}_{3}=e_{4} ; \\
& \dot{e}_{4}=-\frac{u_{\delta}}{N}+\eta(t) .
\end{aligned}
$$

Since variables $x_{1 \delta}$ and $x_{2 \delta}$ are available, then, from Remark 1 , the flat outputs $F_{\delta}=x_{1 \delta}+x_{2 \delta}$ and $F_{\delta}^{(2)}=-x_{2 \delta}$ are also available, implying that the tracking errors $e_{1}=F_{\delta}-F_{*}$ and $e_{3}=F_{\delta}^{(2)}-F_{*}^{(2)}$ can be measured simultaneously. Hence, a set of decoupled high-gain extended linear Luenberger observers for the estimation of the unknown variables $\left\{e_{2}, e_{4}, \eta\right\}$ is proposed as follows:

$$
\begin{aligned}
& \dot{\hat{e}}_{1}=\widehat{e}_{2}+2 w_{0}\left(e_{1}-\widehat{e}_{1}\right) ; \\
& \dot{\hat{e}}_{2}=e_{3}+w_{0}^{2}\left(e_{1}-\widehat{e}_{1}\right) ; \\
& \dot{\hat{e}}_{3}=\widehat{e}_{4}+3 w_{0}\left(e_{3}-\widehat{e}_{3}\right) ; \\
& \dot{\hat{e}}_{4}=-\frac{u_{\delta}}{N}+\widehat{\eta}+3 w_{0}^{2}\left(e_{3}-\widehat{e}_{3}\right) ; \\
& \dot{\hat{\eta}}=w_{0}^{3}\left(e_{3}-\widehat{e}_{3}\right),
\end{aligned}
$$

where $w_{0}>1$. Notice that the above linear observers are in agreement with the EOS proposed in (26) and (28). Having proposed the high-gain observers for the estimation of the unknown variables, we proceed to design the linear controller based on the ADRC approach. That is, the input $u_{\delta}$ will be synthesized with an active disturbance canceling strategy for the uncertain $\eta$, in terms of its estimated value $\hat{\eta}$, and the use of variables $\widehat{e}_{2}$ and $\widehat{e}_{4}$ instead of the actual variables $e_{2}$ and $e_{4}$, respectively. Therefore, according to (18) and (31), we can propose $u_{\delta}$ as

$$
u_{\delta}=N\left(k_{4} \widehat{e}_{4}+k_{3} e_{3}+k_{2} \widehat{e}_{2}+k_{1} e_{1}-F_{*}^{(4)}+\widehat{\eta}\right),
$$


where $K^{T}=\left[k_{1}, k_{2}, k_{3}, k_{4}\right]$ must be selected, such that the matrix

$$
H_{4}=\left[\begin{array}{rrrr}
0 & 1 & 0 & 0 \\
0 & 0 & 1 & 0 \\
0 & 0 & 0 & 1 \\
-k_{1} & -k_{2} & -k_{3} & -k_{4}
\end{array}\right]
$$

is Hurwitz (see definition in (32)).

Remark 3. Matrix $\underline{A}$ is defined by

$$
\underline{A}=\left[\begin{array}{lll}
-3 & 1 & 0 \\
-3 & 0 & 1 \\
-1 & 0 & 0
\end{array}\right]
$$

(see (30)), with $\lambda_{j}\{\underline{A}\}=-1$ and $j=\{1,2,3\}$. On the other hand, the first and second equations of (38) are neglected, because the observation error $\widetilde{e}_{1}=e_{1}-\widehat{e}_{1}$ satisfies the following equation:

$$
\ddot{\widetilde{e}}_{1}+2 w_{0} \dot{\widetilde{e}}_{1}+\widetilde{e}_{1}=0 .
$$

This implies that $\tilde{e}_{2}$ exponentially converges to zero.

From the discussion above, the following can be concluded.

Fact 1. $\eta(t)$, defined in (35), satisfies the conditions in Assumption (A1).

Fact 2. Selecting, for instance, $K^{T}$, such that $\lambda_{j}\left\{H_{4}\right\}=-1$ for $i=\{1,2,3,4\}$ and $w_{0} \gg 1$, we can always fulfill the conditions in Assumptions (A2) and (A3).

From Facts 1 and 2 and according to Theorem 2, we can assure that the system locally asymptotically and exponentially converges to zero, if $F_{*}=0$. Besides, if $F_{*}(t) \neq 0$ and its time derivatives up to order 4 are bounded, then we can always assure that the tracking error is ultimately bounded, where the confined region of the tracking error can be as small as desired.

Remark 4. In order to avoid the large initial peaking phenomena found in the response of observer variables, we suggest using a clutch function to smooth these transient peaking responses in all the observer variables used in the controller. The "clutch" is defined as a time function smoothly increasing from 0 to 1 , during a small time interval $[0, \alpha)$. We fix the smoothing function as

$$
s_{f}(t)= \begin{cases}1 & \text { for } t>\alpha ; \\ \sin ^{r}\left(\frac{\pi t}{2 \alpha}\right) & \text { for } 0 \leq t<\alpha,\end{cases}
$$

where $r$ is a suitably large positive even integer (see [19]). Therefore, the "smoothing" of the observer variables may be implemented as

$$
\widehat{z}_{f}=\widehat{z} s_{f}(t)
$$

where $z=\left\{e_{2}, e_{4}, \eta\right\}$.
Remark 5. As already mentioned, an advantage of combining the flatness and the ADRC methods is that it allows online estimates and cancels the undesirable effects of the higherorder nonlinearities discarded by the linearization approximation. Because our result is based on this advantage, it is important to provide some arguments to validate it. The ADRC is based on restriction to (1) flat systems and their corresponding input-to-flat outputs dynamics (multivariable or monovariable) and (2) a brute force exact linearization of the underlying input output dynamics (monovariable) or the set of statically or dynamically decoupled nonlinear dynamics, written in Isidori's canonical form. This is achieved by online estimating and online feedback cancelling of absolutely everything that perturbs the forced linear dynamics from its desired nominal behaviour (trajectory tracking or stabilization). This uncertainty cancelling includes poorly known additive expressions containing state-dependent nonlinearities and the unpredictable effects of unmodeled dynamics and of external unknown disturbances. Very many examples and applications regarding the effectiveness of this technique as well as the theoretical results backing the methodology actually constitute sufficient proof of the assertion made in Abstract of the paper. References [13-19] are vivid proof of the range of applications and effectiveness of the method, not to mention the underlying simplicity. The following articles also contain sufficient results and applications which validate the technique in a generous manner [32-36]. Reference [37] offers complete survey up to 2014.

Numerical Simulation. To test the performance of the proposed ADRC in conjunction with the flatness controller, we carried out two numerical simulations, with the following setup:

$$
\begin{aligned}
m & =0.1 \mathrm{Kg} ; \\
M & =0.5 \mathrm{Kg} ; \\
L_{r} & =0.5 \mathrm{~m} ; \\
L & =0.5 \mathrm{~m} ; \\
I_{b} & =0.13 \mathrm{Kgm}^{2},
\end{aligned}
$$

and the closed loop characteristic polynomial chosen as

$$
p(s)=\left(s^{2}+2 \zeta \omega_{n} s+\omega_{n}^{2}\right)^{2},
$$

with $\zeta=0.707$ and $\omega_{n}=1.2$. Notice that $I_{b}$ was computed via the formula $I_{b}=M\left(L_{r}^{2}+L^{2} / 12\right)$. The aim of the first simulation was to solve the stabilization of the BRT system at the rest equilibrium position. The other simulation solved the output feedback trajectory control. To this end, we use the control equation, (39), with the decoupled corresponding observer (38). The observer parameter was fixed as $w_{0}=10$, and the clutch parameters were set as $\alpha=0.1[\mathrm{~s}]$ and $r=3$.

The Output Feedback Stabilizing Controller. The closed loop stabilization to the GPI observer based on the ADRC of the system (8) was carried out. To this end, the reference trajectory, $F_{*}$, for the flat output, $F_{\delta}(t)$, was set to zero, 

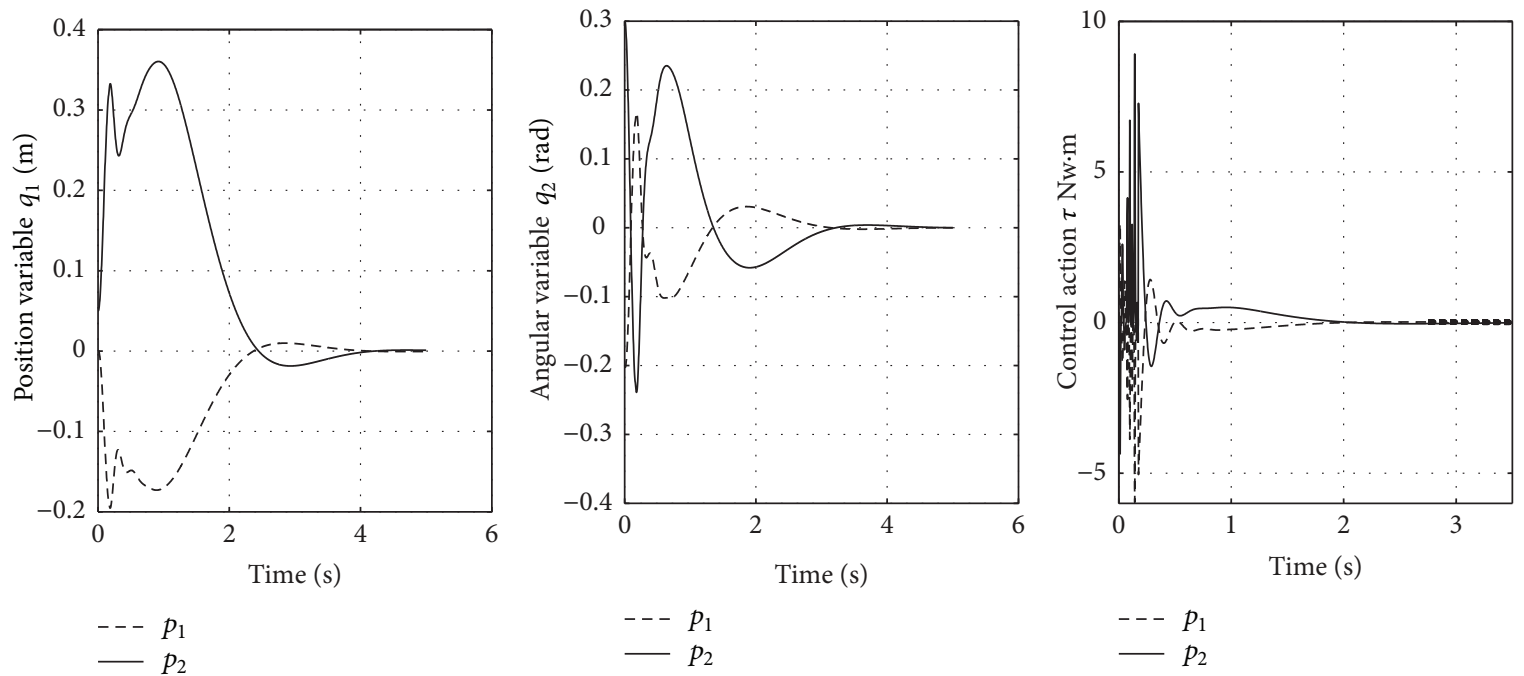

FIGURE 2: Closed loop response of the ADRC for two different initial conditions: $p_{1}$ and $p_{2}$.

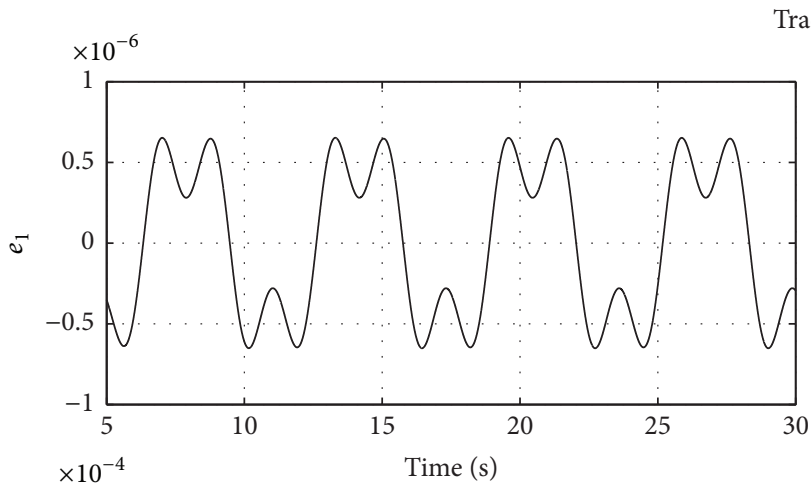

Tracking errors
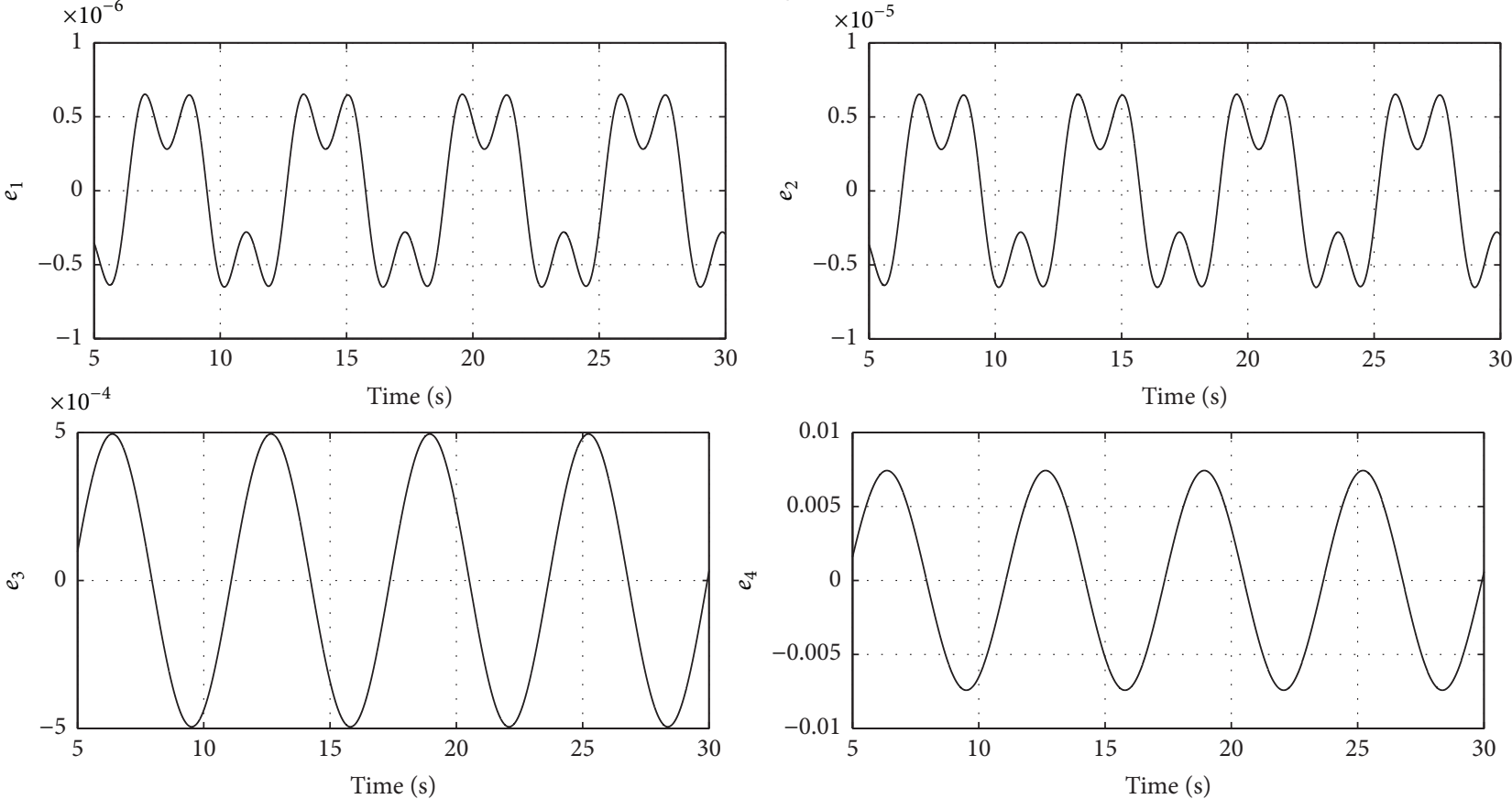

FIgURE 3: A window time, from 5 to 30 seconds, of the tracking trajectory error when the reference is a sinusoidal signal.

as required by the stabilization task. The pair of initial conditions, for equal numbers of executions, was selected as

$$
\begin{aligned}
p_{1} & =\left(x_{1}=0, \dot{x}_{1}=0.5[\mathrm{~m} / \mathrm{s}], x_{2}=-0.2[\mathrm{rad}], \dot{x}_{2}\right. \\
& =-0.1[\mathrm{rad} / \mathrm{s}]), \\
p_{2} & =\left(x_{1}=0.1[\mathrm{~m}], \dot{x}_{1}=0.3[\mathrm{~m} / \mathrm{s}], x_{2}=0, \dot{x}_{2}\right. \\
& =-0.1) .
\end{aligned}
$$

Figure 2 shows the closed loop responses of the ADRC for the stabilization of system (8) at the rest position for each initial condition. From this figure we can see that, before 4 seconds elapse, the position variables and the control action go to zero.

The Output Feedback Trajectory Tracking Problem. A more challenging control task was selected for the second experiment, where the sine signal $F_{*}(t)=0.4 \sin (t / 2)$ was selected as the reference trajectory. To this end, we fixed the initial condition at the origin and used the same setup as in the previous simulation.

Figure 3 shows a window from 5 to 30 seconds of the evolution of the tracking errors $e_{i}=F_{\delta}^{(i-1)}-F_{*}^{(i-1)}(t)$, with $i=\{1,2,3,4\}$. The tracking errors $e_{1}, e_{2}, e_{3}$, and $e_{4}$, are, 

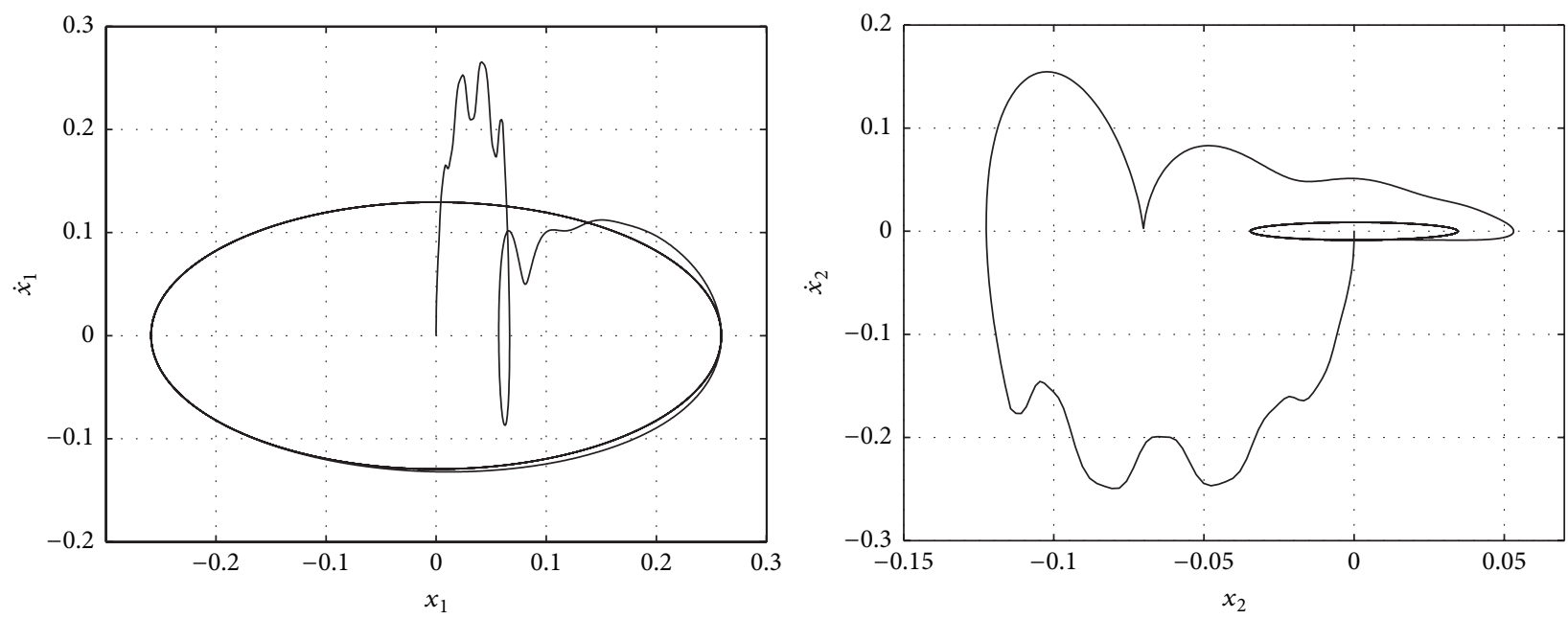

FIgURE 4: Phase space of the position and angular variables.

respectively, in the ranges of $10^{-6}, 10^{-5}, 10^{-4}$, and $10^{-3}$. From these results we can claim that the system is able to effectively track the proposed reference after 4 seconds has elapsed. Additionally, the closed loop response showed a considerably large domain of attraction.

Finally, Figure 4 shows the phase space of the position and angular variables. There, we can see that those variables have a limit cycle in their corresponding phase spaces.

\section{Conclusions}

The differential flatness approach, in conjunction with ADRC, allows systematic solutions to a number of interesting nonlinear control problems. In this instance, we have exploited the local flatness property for the efficient stabilization and tracking of the underactuated BRT. It is important to emphasize that this system is not feedback-linearizable and that its relative degree is not well defined. Moreover, as far as we know, the stabilization of the BRT remained unsolved by using either the shaping energy approach or the IDA-PBC method. Nevertheless, this problem can be partially solved using linear control theory. The fact that the tangent linearization of this system, around the unstable equilibrium point, is locally controllable implies that the system is also locally flat. This allows us to use the robust ADRC in the efficient online estimation of the locally neglected nonlinearities and their active feedback cancelation. The solution, which is quite robust with respect to unmodeled disturbances and neglected nonlinearities, is, in fact, a linear controller with an online compensator. It is based on a set of decoupled linear extended observers and a single linear output feedback controller, with disturbance cancelation features. The proposed controller guarantees locally exponentially asymptotic stability for the stabilization problem and practical and local stability in the solution of the tracking error. To assess the effectiveness of the proposed methodology, numerical simulations were carried out. From the simulation results, we can demonstrate that the proposed controller behaves remarkably well, having an acceptable domain of attraction.

\section{Appendix}

\section{Proof of Theorem 2}

The following proof is based on the previous works [3840]. First of all, we define the actual extended state $\mathbf{x}_{e}=$ $\left[x_{1}, x_{2}, \ldots, x_{n}, f\right]^{T} \in \mathbb{R}^{n+1}$. Hence, system (25) can be rewritten as

$$
\begin{aligned}
& \dot{\mathbf{x}}_{e}=A_{n+1} \mathbf{x}_{e}+B_{n+1} u+E_{n+1} \eta ; \\
& y_{e}=C_{n+1}^{T} \mathbf{x}_{e},
\end{aligned}
$$

with $\eta:=\dot{f}$. Evidently, system (26) is a Luenberger observer for the system above. Defining the observation error vector as $\widetilde{\mathbf{x}}_{e}=\mathbf{x}_{e}-\widehat{\mathbf{x}}_{e}$, we can easily obtain, from (A.1) and (26), the following equation:

$$
\dot{\widetilde{\mathbf{x}}}_{e}=\left(A_{n+1}-l C_{n+1}^{T}\right) \widetilde{\mathbf{x}}_{e}+E_{n+1} \eta
$$

which is equivalent to

$$
\dot{\xi}=w_{0} \underline{A} \xi+w_{0}^{-(n+1)} E_{n+1} \eta,
$$

where we use the following transformation:

$$
\widetilde{\mathbf{x}}_{e}:=\operatorname{diag}\left\{w_{0}, w_{0}^{2}, \ldots, w_{0}^{n+1}\right\} \xi=W_{n+1} \xi .
$$

Using the definition of $\eta=\dot{f}$ and (33), we obtain

$$
\begin{aligned}
\dot{\xi}= & w_{0} \underline{A} \xi+w_{0}^{-(n+1)} \frac{\partial f}{\partial x_{n}} \\
& \cdot E_{n+1}\left(\left(\nabla_{\mathbf{x}} f A_{n}-K^{T}\right) \mathbf{e}+\bar{K}^{T} W_{n+1} \xi\right) \\
+ & w_{0}^{-(n+1)} E_{n+1} \Delta(t),
\end{aligned}
$$

where the definition of $\underline{A}$ was defined in (30).

Stability Analysis of the Trajectory Tracking Error $\mathbf{e}=\mathbf{x}-\mathbf{y}_{r}$. Substituting the value of (31) in (25) leads to

$$
\dot{\mathbf{x}}=A_{n} \mathbf{x}+E_{n}\left(-K^{T}\left(\widehat{\mathbf{x}}-\mathbf{y}_{r}\right)+w_{0}^{n+1} \xi_{n+1}+y_{r}^{(n)}\right) .
$$


Expressing the last equation in terms of the tracking error, we obtain

$$
\dot{\mathbf{e}}=H_{n} \mathbf{e}+E_{n} \bar{K}^{T} W_{n+1} \xi .
$$

where $\bar{K}^{T}=\left[\begin{array}{ll}K^{T} & 1\end{array}\right]$ and $H_{n}=A_{n}-E_{n} K^{T}$. Now, we analyse the convergence of (A.7) and (A.5). Then, both equations are easily rewritten as

$$
\left[\begin{array}{c}
\dot{\mathbf{e}} \\
\dot{\xi}
\end{array}\right]=\left[\begin{array}{cc}
H_{n} & E_{n} \bar{K}^{T} W_{n+1} \\
0 & w_{0} \underline{A}
\end{array}\right]\left[\begin{array}{l}
\mathbf{e} \\
\xi
\end{array}\right]+\left[\begin{array}{c}
0_{n \times 1} \\
\frac{E_{n+1}}{w_{0}^{n+1}}
\end{array}\right] \Phi(\cdot),
$$

where

$$
\begin{aligned}
\Phi(\mathbf{x}, \mathbf{e}, \xi, t) & =F_{1}(\mathbf{x}) \mathbf{e}+F_{2}(\mathbf{x}) \xi+\Delta(t) ; \\
F_{1}(\cdot) & =\frac{\partial f}{\partial x_{n}}\left(\nabla_{\mathbf{x}} f A_{n}-K^{T}\right) ; \\
F_{2}(\cdot) & =\frac{\partial f}{\partial x_{n}} \bar{K}^{T} W_{n+1} .
\end{aligned}
$$

Hence, $\Phi(\cdot)$ is continuous and bounded, according to Assumption (A1). Introduce the following change of coordinates:

$$
\zeta=\mathbf{e}+N \xi
$$

where $N$ is a constant matrix, which is a solution of the following Sylvester equation:

$$
E_{n} \bar{K}^{T} W_{n+1}=H_{n} N-w_{0} N \underline{A} .
$$

Assumption (A2) assures existence and uniqueness of $N$ [41]. Consequently, (A.10) can be rewritten as

$$
\left[\begin{array}{l}
\dot{\zeta} \\
\dot{\xi}
\end{array}\right]=\left[\begin{array}{cc}
H_{n} & 0 \\
0 & w_{0} \underline{A}
\end{array}\right]\left[\begin{array}{l}
\zeta \\
\xi
\end{array}\right]+\left[\begin{array}{c}
0_{n \times 1} \\
\frac{E_{n+1}}{w_{0}^{n+1}}
\end{array}\right] \Phi(\cdot) .
$$

Because $H_{n}$ is selected to be Hurwitz, we conclude that $\zeta$ converges exponentially to zero. Finally, we need to prove boundedness and convergence of the system:

$$
\dot{\xi}=w_{0} \underline{A} \xi+w_{0}^{-(n+1)} E_{n+1} \Phi(\mathbf{x}, 0, \xi, t) .
$$

To this end, we need to introduce the following auxiliary lemma to estimate the norm of the matrix $N$, without the need to compute it.

Lemma A.1. Under Assumptions (A2) and (A3), the matrix $N$ of (A.11) is bounded by

$$
\|N\| \leq w_{0}^{n} k_{N}, \quad k_{N}:=\frac{w_{0}\|\bar{K}\|\left\|\underline{A}^{-1}\right\|}{w_{0}-\left\|H_{n}\right\|\left\|\underline{A}^{-1}\right\|}>0 .
$$

Besides, for $\bar{K}$ that is sufficiently large, then $\lim _{\|K\| \rightarrow \infty}\|N\| \leq$ $w_{0}^{n+1}$; because the proof of this fact is not difficult, it is omitted.
Resuming the stability proof, because $\underline{A}$ is Hurwitz, there exists $P=P^{T}>0$, such that $P \underline{A}+\underline{A}^{T} P=-2 I_{n+1}$. Defining the Lyapunov function $V=(1 / 2) \xi^{T} \overline{P \xi}$, it is easy to see that its time derivative, along the trajectories of (A.13), can be upper bounded by

$$
\begin{aligned}
\dot{V} \leq & -w_{0}|\xi|^{2}+\lambda_{\max }(P)\left(\frac{k_{F_{1}} k_{N}}{w_{0}}+\frac{k_{F_{2}}}{w_{0}^{(n+1)}}\right)|\xi|^{2} \\
& +\frac{\lambda_{\max }(P) k_{\Delta}|\xi|}{w_{0}^{(n+1)}}
\end{aligned}
$$

where $|\Delta| \leq k_{\Delta},\left\|F_{1}\right\| \leq k_{F_{1}}$, and $\left\|F_{2}\right\| \leq k_{F_{2}}$. From the above, we need to analyse two cases:

$\left(k_{\Delta}=0\right)$ Selecting $w_{0}>1$, such that

$$
\lambda_{\max }(P)\left(\frac{k_{F_{1}} k_{N}}{w_{0}}+\frac{k_{F_{2}}}{w_{0}^{n+1}}\right)=k_{\xi}<w_{0},
$$

then $\dot{V}<0$, concluding that $\xi$ exponentially converges to zero. Notice that (A.16) can be always satisfied selecting the free observer parameter $w_{0} \gg 1$.

$\left(k_{\Delta} \neq 0\right)$ If (A.16) holds, then from (A.15) the ultimate boundedness of $\|\xi\|$ with ultimate bound is

$$
\|\xi\| \leq \frac{\lambda_{\max }(P) k_{\Delta}}{w_{0}^{(n+2)}\left(w_{0}-k_{\xi}\right)} .
$$

Notice that $w_{0}-k_{\xi} \gg 1$, by selecting $w_{0}$ that is sufficiently large. Hence, $\|\xi\| \sim \mathcal{O}\left(1 / w_{0}^{(n+2)}\right)$. On the other hand, from (A.10), since $\|\zeta\| \rightarrow 0$, the error $\|\mathbf{e}\| \sim \mathcal{O}\left(1 / w_{0}^{2}\right)$. That is, the conditions of Theorem 2 are satisfied.

\section{Competing Interests}

The authors declare that they have no competing interests.

\section{Acknowledgments}

This research was supported by the Centro de Investigación en Computación of the Instituto Politécnico Nacional (CICIPN) and by the Secretaría de Investigación y Posgrado of the Instituto Politécnico Nacional (SIP-IPN), under Research Grants 20160268 and 20161637. This research was done while Dr. Carlos Aguilar-Ibanez was on sabbatical leave from the Departamento de Mecatrónica del CINVESTAV.

\section{References}

[1] I. Fantoni and R. Lozano, Non-Linear Control for Underactuated Mechanical Systems, Springer, 2002.

[2] I. Sarras, J. Á. Acosta, R. Ortega, and A. D. Mahindrakar, "Constructive immersion and invariance stabilization for a class of underactuated mechanical systems," Automatica, vol. 49, no. 5, pp. 1442-1448, 2013.

[3] S. González-Vázquez and J. Moreno-Valenzuela, "Motion control of a quadrotor aircraft via singular perturbations," International Journal of Advanced Robotic Systems, vol. 10, article 368, 2013. 
[4] C. Aguilar-Avelar and J. Moreno-Valenzuela, "A composite controller for trajectory tracking applied to the Furuta pendulum," ISA Transactions, vol. 57, pp. 286-294, 2015.

[5] M. W. Spong, "Underactuated mechanical systems," in Control Problems in Robotics and Automation, pp. 135-150, Springer, Berlin, Germany, 1998.

[6] R. Olfati-Saber, Nonlinear control of underactuated mechanical systems with application to robotics and aerospace vehicles [Ph.D. thesis], Massachusetts Institute of Technology, 2000.

[7] B. Gao, H. Chen, and X. Zhang, "Control design for underactuated mechanical systems: a survey," Electric Machines and Control, vol. 10, no. 5, p. 541, 2006.

[8] H. Sira-Ramirez, "On the control of the 'ball and beam' system: a trajectory planning approach," in Proceedings of the IEEE Conference on Decision and Control, Sydney, Australia, 2000.

[9] P. Rouchon, M. Fliess, J. Lévine, and P. Martin, "Flatness, motion planning and trailer systems," in Proceedings of the 32nd IEEE Conference on Decision and Control, pp. 2700-2705, IEEE, San Antonio, Tex, USA, December 1993.

[10] M. Fliess, J. Lévine, P. Martin, and P. Rouchon, "Design of trajectory stabilizing feedback for driftless at systems," in Proceedings of the Third ECC, pp. 1882-1887, Rome, Italy, 1995.

[11] J. Lévine, Analysis and Control of Nonlinear Systems: A FlatnessBased Approach, Springer Science \& Business Media, Berlin, Germany, 2009.

[12] M. Fliess, J. Lévine, P. Martin, and P. Rouchon, "Flatness and defect of non-linear systems: introductory theory and examples," International Journal of Control, vol. 61, no. 6, pp. 1327-1361, 1995.

[13] M. Ramírez-Neria, H. Sira-Ramírez, R. Garrido-Moctezuma, and A. Luviano-Juárez, "Linear active disturbance rejection control of underactuated systems: the case of the Furuta pendulum," ISA Transactions, vol. 53, no. 4, pp. 920-928, 2014.

[14] H. Sira-Ramírez, C. López-Uribe, and M. Velasco-Villa, "Linear observer-based active disturbance rejection control of the omnidirectional mobile robot," Asian Journal of Control, vol. 15, no. 1, pp. 51-63, 2013.

[15] J. Han, "From PID to active disturbance rejection control," IEEE Transactions on Industrial Electronics, vol. 56, no. 3, pp. 900906, 2009.

[16] Z. Gao, Y. Huang, and J. Han, "An alternative paradigm for control system design," in Proceedings of the 40th IEEE Conference on Decision and Control (CDC'01), vol. 5, pp. 45784585, Orlando, Fla, USA, December 2001.

[17] Z. Gao, S. Hu, and F. Jiang, "A novel motion control design approach based on active disturbance rejection," in Proceedings of the 40th IEEE Conference on Decision and Control (CDC '01), vol. 5, pp. 4877-4882, Orlando, Fla, USA, December 2001.

[18] R. Li, T. Li, Q. Zheng, and Q. Li, "Ship tracking control based on linear active disturbance rejection control," in Proceedings of the 2012 3rd International Conference on Intelligent Control and Information Processing (ICICIP '12), pp. 201-205, IEEE, Dalian, China, July 2012.

[19] R. Morales, H. Sira-Ramírez, and J. A. Somolinos, "Linear active disturbance rejection control of the hovercraft vessel model," Ocean Engineering, vol. 96, pp. 100-108, 2015.

[20] H. Coral-Enriquez, J. Cortés-Romero, and G. A. Ramos, "Robust active disturbance rejection control approach to maximize energy capture in variable-speed wind turbines," Mathematical Problems in Engineering, vol. 2013, Article ID 396740, 12 pages, 2013.
[21] J. Cortés-Romero, H. Rojas-Cubides, H. Coral-Enriquez, H. Sira-Ramírez, and A. Luviano-Juárez, "Active disturbance rejection approach for robust fault-tolerant control via observer assisted sliding mode control," Mathematical Problems in Engineering, vol. 2013, Article ID 609523, 12 pages, 2013.

[22] R. Li, T. Li, R. Bu, Q. Zheng, and C. L. P. Chen, "Active disturbance rejection with sliding mode control based course and path following for underactuated ships," Mathematical Problems in Engineering, vol. 2013, Article ID 743716, 9 pages, 2013.

[23] H. K. Khalil, "High-gain observers in nonlinear feedback control," in Proceedings of the International Conference on Control, Automation and Systems (ICCAS '08), pp. 47-57, IEEE, Seoul, South Korea, October 2008.

[24] S. Puga, M. Bonilla, M. Malabre, and R. Lozano, "Singularly perturbed implicit control law for linear time varying SISO systems," International Journal of Robust and Nonlinear Control, vol. 24, no. 10, pp. 1530-1549, 2014.

[25] S. Puga, M. Bonilla, C. H. Moog, M. Malabre, and R. Lozano, "Singularly perturbed feedback linearization for SISO nonlinear systems with measurement of the state," in Proceedings of the European Control Conference (ECC '15), pp. 3250-3255, Linz, Austria, July 2015.

[26] J. D. J. Rubio, G. Ochoa, R. Balcazar, and J. Pacheco, "Uniform stable observer for the disturbance estimation in two renewable energy systems," ISA Transactions, vol. 58, pp. 155-164, 2015.

[27] A. M. Bloch, N. E. Leonard, and J. E. Marsden, "Matching and stabilization by the method of controlled lagrangians," in Proceedings of the 37th IEEE Conference on Decision and Control, vol. 2, pp. 1446-1451, IEEE, Piscataway, NJ, USA, 1998.

[28] R. Ortega, M. W. Spong, F. Gómez-Estern, and G. Blankenstein, "Stabilization of a class of underactuated mechanical systems via interconnection and damping assignment," IEEE Transactions on Automatic Control, vol. 47, no. 8, pp. 1218-1233, 2002.

[29] R. Ortega and E. Garcia-Canseco, "Interconnection and damping assignment passivity-based control: a survey," European Journal of Control, vol. 10, no. 5, pp. 432-450, 2004.

[30] Q. Gao, Y. Hou, K. Li, Z. Sun, C. Wang, and R. Hou, "Neural network based active disturbance rejection control of a novel electrohydraulic servo system for simultaneously balancing and positioning by isoactuation configuration," Shock and Vibration, vol. 2016, Article ID 4921095, 9 pages, 2016.

[31] S. Galvan-Colmenares, M. A. Moreno-Armendáriz,, J. de Jesús Rubio, F. Ortíz-Rodriguez, W. Yu, and C. F. Aguilar-Ibáñez, "Dual PD control regulation with nonlinear compensation for a ball and plate system," Mathematical Problems in Engineering, vol. 2014, Article ID 894209, 10 pages, 2014.

[32] Q. Zheng, L. Q. Gao, and Z. Gao, "On validation of extended state observer through analysis and experimentation," Journal of Dynamic Systems, Measurement and Control, vol. 134, no. 2, Article ID 024505, 2012.

[33] B.-Z. Guo and Z.-L. Zhao, "Weak convergence of nonlinear high-gain tracking differentiator," IEEE Transactions on Automatic Control, vol. 58, no. 4, pp. 1074-1080, 2013.

[34] B.-Z. Guo and Z.-L. Zhao, "On the convergence of an extended state observer for nonlinear systems with uncertainty," Systems \& Control Letters, vol. 60, no. 6, pp. 420-430, 2011.

[35] B.-Z. Guo and Z.-L. Zhao, "On convergence of the nonlinear active disturbance rejection control for MIMO systems," SIAM Journal on Control and Optimization, vol. 51, no. 2, pp. 1727-1757, 2013. 
[36] W.-H. Chen, J. Yang, L. Guo, and S. Li, "Disturbance-observerbased control and related methods-an overview," IEEE Transactions on Industrial Electronics, vol. 63, no. 2, pp. 1083-1095, 2016.

[37] R. Madoński and P. Herman, "Survey on methods of increasing the efficiency of extended state disturbance observers," ISA Transactions, vol. 56, pp. 18-27, 2015.

[38] A. N. Atassi and H. K. Khalil, "A separation principle for the stabilization of a class of nonlinear systems," IEEE Transactions on Automatic Control, vol. 44, no. 9, pp. 1672-1687, 1999.

[39] W. Zhou, S. Shao, and Z. Gao, "A stability study of the active disturbance rejection control problem by a singular perturbation approach," Applied Mathematical Sciences, vol. 3, no. 9-12, pp. 491-508, 2009.

[40] H. K. Khalil and L. Praly, "High-gain observers in nonlinear feedback control," International Journal of Robust and Nonlinear Control, vol. 24, no. 6, pp. 993-1015, 2014.

[41] R. Bhatia and P. Rosenthal, "How and why to solve the operator equation $\mathrm{AX}-\mathrm{XB}=Y$," The Bulletin of the London Mathematical Society, vol. 29, no. 1, pp. 1-21, 1997. 


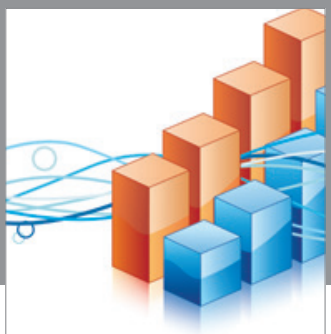

Advances in

Operations Research

vatem alat4

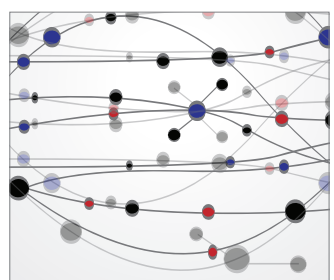

\section{The Scientific} World Journal
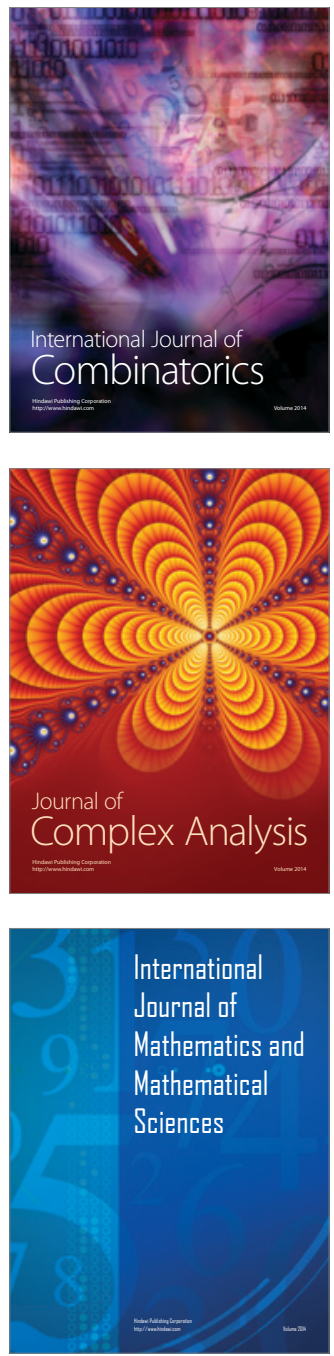
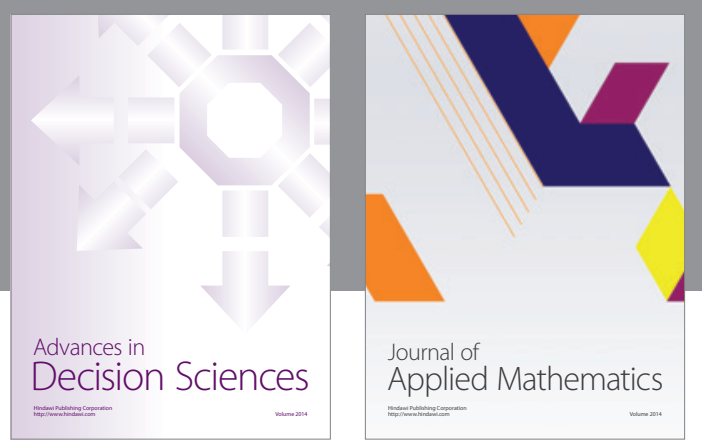

Algebra

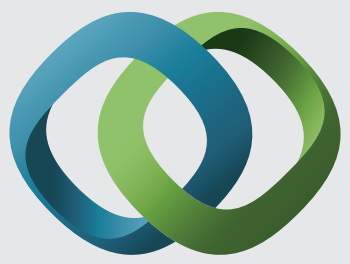

\section{Hindawi}

Submit your manuscripts at

http://www.hindawi.com
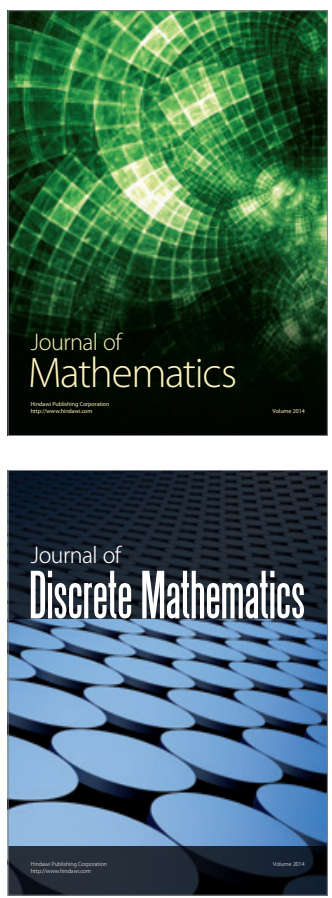

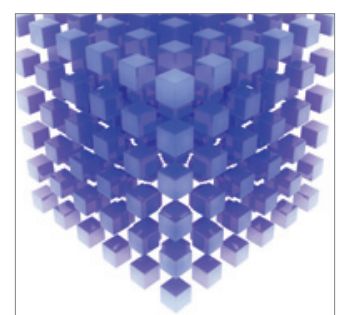

Mathematical Problems in Engineering
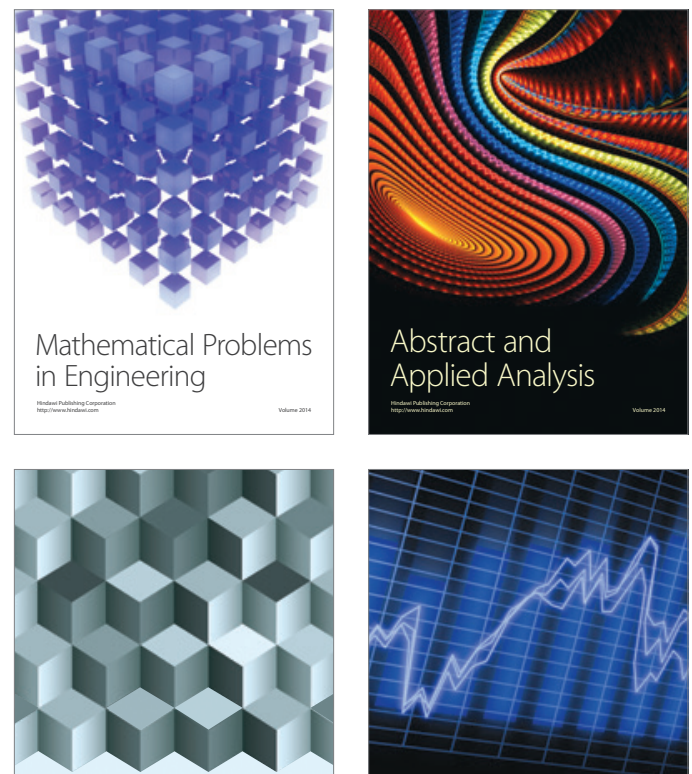

Journal of

Function Spaces

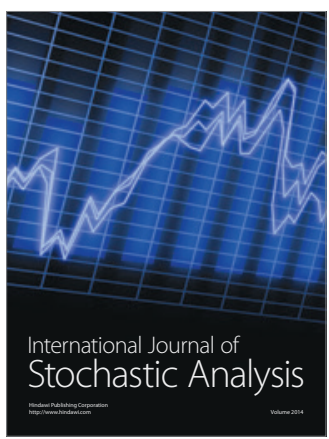

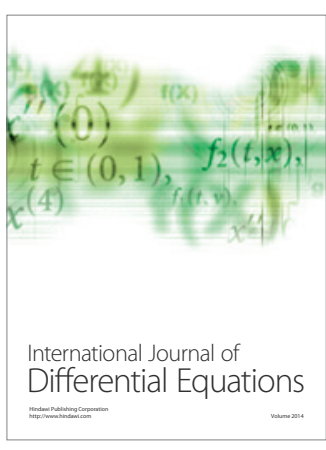
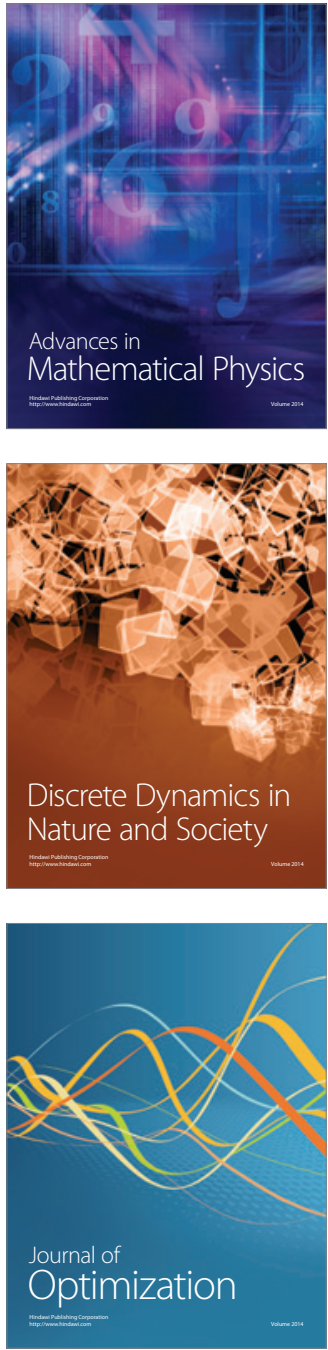